\title{
Preoperative Correction of Tc-99-m-MIBI SPECT by F18_FDG Cardiac PET/CT for Myocardial Viability Before CABG
}

\author{
(D) Süreyya Talay1, (1) Nahide Belgit Talay²
}

129 Mayis State Hospital, Cinic of Cardiovascular Surgery, Ankara, Turkey

${ }^{2}$ Çanakkale Mehmet Akif Ersoy State Hospital, Clinic of Nuclear Medicine, Çanakkale, Turkey

\begin{abstract}
Objectives: Coronary artery bypass graft (CABG) surgery has been safely practiced on cases with poor left ventricular ejection fraction (LVEF). Although adversely effects of surgery with higher mortality and morbidity rates, preoperative myocardial viability defines outcome. Thus, it is crucial to determine preoperative left ventricular myocardial tissue viability for acceptable postoperative results in this group of patients. This multi-center prospective study focuses of assessment of myocardial perfusion and viability by comparison of two different nuclear medicine imaging techniques; technetium 99-m-methoxy-isobutyl-isonitrile single photon emission computed tomography (Technetium99-m-MIBI SPECT) and cardiac fluorodeoxyglucose positron emission tomography-computed tomography (F18- FDG Cardiac PET/CT).
\end{abstract}

Materials and Methods: Study is performed by ethical approval and Helsinki protocols. Each patient was evaluated by Technetium-99-m-MIBI SPECT and $\mathrm{F}^{18}$ FDG Cardiac PET/CT for myocardial viability detection prior to CABG surgery. Technetium-99-m-MIBI SPECT and $\mathrm{F}^{18}$-FDG Cardiac PET/CT was evaluated in 191 cases

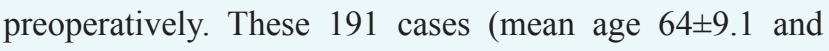
male $\mathrm{n}=103,53.9 \%$ ) with LVEF $\leq 35 \%$ were evaluated for coronary revascularization preoperatively.

Results: Study data accumulated from cases of CABG $(\mathrm{n}=191)$ with preoperative LVEF measurements between $35 \%$ to $20 \%(n=154,80.6 \%)$ and lower than $20 \%(n=37$, 19.3\%). Technetium-99-m-MIBI SPECT imaging was performed before $\mathrm{F}^{18}$-FDG Cardiac PET/CT in all cases. By these evaluations, 1896 segments were detailed in total. For Technetium-99-m-MIBI SPECT, 1036 segments displayed normal Technetium-99-m-MIBI uptake ( $\geq 70 \%$, classification 2). On the other hand, 860 segments displayed reduced Technetium-99-m-MIBI uptake with

Address for Correspondence: Süreyya Talay, 29 Mayıs State Hospital, Cinic of Cardiovascular Surgery, Ankara, Turkey e-mail: suereyyatalay@gmail.com ORCID: orcid.org/0000-0002-4908-3850

Received: 05.12.2019 Accepted: 20.02.2020

Cite this article as: Talay S, Talay NB. Preoperative correction of Tc-99-m-MIBI SPECT by F18-FDG Cardiac PET/CT for Myocardial Viability Before CABG. EJCM 2020;8(1):10-20.

DOI: 10.32596/ejcm.galenos.2020.12.060

${ }^{\circ}$ Copyright 2020 by Heart and Health Foundation of Turkey (TÜSAV) / E Journal of Cardiovascular Medicine published by Galenos Publishing House. 


\section{Abstract}

different degrees $(<70 \%)$. Elaborations of the latter data, scar tissue with absent Technetium-99-m-MIBI uptake $(\leq 30 \%)$ was imaged in $441(23.2 \%$, classification 0$)$, reduced Technetium-99-m-MIBI uptake from 30\% to $69 \%$ in $419(22 \%$, classification 1). One thousand and thirty-six segments with normal Technetium-99-m-MIBI uptake $(\geq 70 \%)$ revealed myocardial viability by $\mathrm{F}^{18}$ FDG C-PET/CT in 1036 segments. Predictive value for viability of Technetium-99-m-MIBI SPECT therefore presents a value in $100 \%$ in this group. On the other end of the SPECT images, 441 cases with Technetium-99-mMIBI uptake $\leq 30 \%$ (which otherwise would be accepted as poor candidates for revascularization) presented viability with FDG uptakes in 285 cases. One hundred and fiftysix segments from the latter group were verified by FDG uptake as nonviable. The predictive value for Technetium99-m-MIBI SPECT for this group on myocardial viability presents a rate in $35.3 \%$. Regression analysis revealed a

\section{Introduction}

Coronary artery bypass graft (CABG) surgery is safely performed in cases with poor left ventricular ejection fraction (LVEF). Although it adversely affects high mortality and morbidity rates, preoperative myocardial viability defines outcome. Thus, it is crucial to determine preoperative left ventricular myocardial tissue viability for acceptable postoperative results in this group of patients.

This multi-center prospective study focuses on the assessment of myocardial perfusion and viability by comparison of two different nuclear medicine imaging techniques; technetium 99-m-methoxy-isobutyl-isonitrile single photon emission computed tomography (Technetium99-m-MIBI SPECT) and cardiac fluorodeoxyglucose positron emission tomography-computed tomography (F ${ }^{18}$-FDG Cardiac PET/CT).

\section{Materials and Methods}

Each patient was evaluated by Technetium-99-m-MIBI SPECT and $\mathrm{F}^{18}$-FDG Cardiac PET/CT for myocardial weak linear correlation between segmental $\mathrm{F}^{18}$-FDG and Tc-99-m-MIBI uptake with a correlation coefficient of 0.30 $(n=441, p<0.05)$.

Conclusion: Prior to coronary artery bypass graft surgery decision for cases with reduced preoperative left ventricular ejection fraction, cardiac PET/CT using $\mathrm{F}^{18}$-FDG predicts myocardial viability more effectively when compared with Tc-99-m-MIBI SPECT alone. After a report of a severe hypoperfusion by Tc-99-m-MIBI SPECT analyse, it is suggestible to applicate to $\mathrm{F}^{18}$-FDG cardiac PET/ $\mathrm{CT}$ as a last resort. The latter technique is more accurate at elaborating and corroborating myocardial viability. We believe that avoiding from surgery by Tc-99-m-MIBI SPECT is a premature decision.

Keywords: Technetium-99m-MIBI SPECT, $\mathrm{F}^{18}$ cardiac FDG PET/CT, myocardial viability, CABG, left ventricular dysfunction

viability detection prior to $\mathrm{CABG}$ surgery. This study was performed in accordance with Helsinki protocols.

\section{Patients}

One hundred and ninety-one cases (mean age 64.9 years and male ratio $n=103,53.9 \%$ ) with $L V E F \leq 35 \%$ were preoperatively evaluated for coronary revascularization by Technetium-99-m-MIBI SPECT and $\mathrm{F}^{18}$-FDG Cardiac $\mathrm{PET} / \mathrm{CT}$. These operations' ratio was $4.4 \%$ in total 4320 $\mathrm{CABG}$ cases. The mean value for calculated preoperative LVEF was $23.5 \pm 8.1$ (range: $11 \%$ to $35 \%$ ).

Indications for $\mathrm{CABG}$ were based on the American Heart Association criteria for coronary stenosis. Degree of LVEF deterioration was not accepted as a contraindication at any level for surgery with demonstrable myocardial viability. On the contrary, non-demonstrable myocardial viability on $\mathrm{F}^{18}$-FDG C-PET/CT despite the existence or absence of myocardial perfusion by Technetium-99-mMIBI SPECT was recognized as a certain contraindication for surgery. 
Functional capacity by the New York Heart Association criteria was defined as class II, III and IV for our study group in percentages as $21(10.9 \%), 81(42.4 \%)$ and 81 (42.4\%), respectively. None of our patients was class I.

Every patient reported a myocardial infarction (MI). Furthermore, 104 (54.4\%) patients reported a percutaneous coronary intervention.

Comorbidities were chronic obstructive pulmonary disease $(\mathrm{n}=35,18.3 \%)$, Diabetes Mellitus $(\mathrm{n}=58,30.3 \%)$ and end-stage renal disease (ESRD) with hemodialysis $(n=29)$.

Emergency cases, concomitant cardiac surgery with valve repair and/or aneurysmectomy were excluded from the study. Thus, we refined the study population to be able to focus on myocardial perfusion and viability detection by Technetium-99-m-MIBI SPECT and $\mathrm{F}^{18}$-FDG Cardiac $\mathrm{PET} / \mathrm{CT}$.

\section{Imaging and Data Evaluation}

Primarily, surgical decision was grounded on coronary angiography with the correlation of myocardial viability evidence from nuclear imaging.

Both Technetium-99-m-MIBI SPECT and $\mathrm{F}^{18}$ FDG Cardiac PET/CT were not performed on the same day. PET/CT was planned the next day for each case. Antianginal medications were ceased prior to nuclear study. Elaboration of images were in identical pixel sizes for both techniques of MIBI SPECT and PET/CT. Diagnostic sensitivity and specifity with MIBI and FDG were obtained and compared using the Fisher's exact test on myocardial viability.

\section{Technetium-99-m-MIBI SPECT}

Each patient received 250 to $400 \mathrm{MBq}$ Technetium99-m injection at rest 1 hour prior to imaging. We used dual double head gamma cameras for transaxial image acquisitions with high resolution collimators (Siemens Corp. Inc. Symbia E-CAM units). Our cut-off frequency was 0.5. A ROI based method was administered for quantitative evaluation. Segmentally highest MIBI accumulation was accepted as a reference line ( $\geq 70 \%)$ for individual segmental perfusion distributions. Thus, visual data for Technetium-99-m-MIBI were reported in three different classifications for segmental perfusion determination; (0): scar tissue without perfusion (infarct, $<30 \%$ ), (1): peri-infarct ischemia with relatively lower perfusion (between 30\% and 70\%), (2): normal perfusion $(\geq 70 \%)$. For segmental analysis of Technetium-99m-MIBI SPECT, we evaluated nine segments by this perfusion classification (anterior, anterolateral, lateral, inferolateral, inferior, inferoseptal, septum, anteroseptal, apex) for each patient and investigation.

\section{F $^{18}$-FDG Cardiac PET/CT}

Patients received $50 \mathrm{~g}$ of glucose orally during resting period 1 hour prior to the investigation. Each patient received 350-500 (10-15 $\mathrm{mCi}) \mathrm{MBq} \mathrm{F} \mathrm{F}^{18}$-FDG injection 30 minutes prior to imaging (Siemens Corp. Inc, model 931108 units). Cut-off frequency was 0.4.

Image analysis and reconstructions for PET/CT were achieved by Apple Comp. Inc. Macintosh Workstation. Afterwards, FDG polar maps were compared with accepted normality modules by means of identifying ventricular segments. Synchronous CT scans were also studied with FDG PET images. A similar ROI based method was also administered for quantitative evaluation. Segmentally highest $\mathrm{F}^{18}$-FDG accumulation was accepted as a reference line ( $\geq 70 \%$ ) for normal viable myocardial tissue. Thus, visual data for $\mathrm{F}^{18}$-FDG uptake was reported in three different classifications for segmental viability; (0): scar tissue without viability (infarct, a ROI calculation value $<50 \%$ ), (1): peri-infarct viability probably hibernating myocardial tissue (a ROI calculation value from $50 \%$ to $70 \%)$, (2): normal viable myocardial tissue ( $\geq 70 \%)$.

For segmental analysis of $\mathrm{F}^{18}$-FDG Cardiac PET/CT, we evaluated anterior, anterolateral, lateral, inferolateral, inferior, inferoseptal, septum, anteroseptal, apex segments for each patient and each investigation.

\section{Surgery}

Coronary artery stenosis $\geq 50 \%$ was generally accepted as a surgical indication. CABG was performed on moderate 
hypothermia with $30{ }^{\circ} \mathrm{C}$ and via cardiopulmonary bypass (CPB). Cardioplegia was applied in three different modules; cold cardioplegia in $10 \mathrm{~mL} /$ per $\mathrm{kg}$ in $40 \mathrm{mmHg}$ pressure, maintaining doses of $500 \mathrm{~mL}$ of normothermic blood cardioplegia in every 20 minutes and $500 \mathrm{~mL}$ before the removal of the cross clamp. Distal anastomoses were performed under cardiac arrest. Patients under 70 years of age were accepted as routine candidates for a left intermamarian artery (LIMA) to left anterior descending artery (LAD). LIMA was used for this position, solely. Proximal anastomoses were performed with a side-biting aortic clamp. No arterial conduit other than LIMA was used.

CPB weaning difficulties and/or arterial hypotension resistant to additive inotropic medications with cardiac index calculation lower than $2.0 \mathrm{~L} /$ minute $/ \mathrm{m}^{2}$ were accepted indications for intra-aortic counterpulsation balloon pump (IABP) implantation.

\section{Statistical Analysis}

Study data were entered into a prospective collection database. The data were expressed as proportions or as mean and standard deviation. Linear regression analysis was administered to calculate the linear correlation of segmental FDG uptake on segmental MIBI uptake. The chi-square analysis was applied for a contingency table to elaborate FDG PET viability detection and MIBI defect severity. P value $\leq 0.05$ was accepted as statistically significant. Statistical modelling was performed using S-PLUS statistical software (MathSoft, Cambridge, MA).

\section{Results}

\section{Perioperative Data}

Study data were accumulated from cases of CABG ( $\mathrm{n}=191)$ with preoperative LVEF measurements between 35 and $20 \%(n=154,80.6 \%)$ and lower than $20 \%(n=37$, $19.3 \%$ ). The mean value for body mass index was $31.4 \pm 4.1$ $\mathrm{kg} / \mathrm{m}^{2}$. Preoperative inotropic agent dependency at the intensive care unit was recorded in 27 cases (14.1\%). Furthermore, 21 cases $(14.1 \%)$ were re-do CABG. The mean values were operation length (281.2 \pm 33.5 minutes), CPB time (152.5 \pm 41.3 minutes), aortic cross clamp time (88.3 \pm 11.4 minutes), intubation length (440.2 \pm 30.3 minutes intensive care unit stay (6.2 \pm 3.1 days), and hospitalization (14.2 \pm 6.2 days). The mean coronary graft number was $3.5 \pm 0.8$. LIMA was used in $179(93.7 \%)$ cases to LAD anastomoses. In each case, preoperative planned revascularization numbers of vessels were anastomosed. We administered IABP in a relatively higher percentage by 81 cases $(42.4 \%)$ via femoral arteries. Forty-three IABPs were placed intraoperatively (in the existence of cardiac function deterioration and lower arterial pressure, we applied IABP before anesthesia induction or with hemodynamical worsening after weaning from CPB ) and 38 cases postoperatively during intensive care unit followups.

Postoperative deaths occurred in 18 cases (9.4\%). Main mortality reasons were longer $\mathrm{CPB}$, postoperative ventricular arrhythmias, postoperative multiple organ deficiencies and major neurological complications. The latter condition was observed at the early hours of intensive care unit. Neurology specialist consultations and brain CT images, when possible, revealed several severe ischemic areas due to central nervous system embolism, which we believe to be originating from aortic plaque removal during aortic manipulations. Postoperative multiple organ deficiencies were severe elevations of hepatic enzymes accompanied by oliguria. Furthermore, ventricular arrhythmia was generally resistant to medication combinations such as lidocaine, amiodaron and/or beta-blockers in these cases. Overall mortality was recorded in 10 cases of re-do CABG operations. Some of these patients were operated in other hospitals and so, it was always possible to determine prior $\mathrm{CABG}$ vessels in this group. Otherwise, medical records were questioned before secondary surgery. Deaths among primary CABG cases were six cases from the LVEF $<20$ group. Other two mortalities were from the patients with higher preoperative LVEF (LVEF between 20 and 35\%). 


\section{Nuclear medicine imaging results}

Technetium-99-m-MIBI SPECT and $\mathrm{F}^{18}$-FDG Cardiac PET/CT were evaluated in 191 cases preoperatively. Recent MIs within 4 weeks were not included to study. Furthermore, the existence of left bundle branch block and severe ischemic mitral deficiencies were also excluded from the study. Previous MI was present in all cases from the study group; 84 anterior wall MI, 64 posterior wall MI and 43 anterior and posterior wall MI.

Technetium-99-m-MIBI SPECT imaging was performed before $\mathrm{F}^{18}$-FDG Cardiac PET/CT in all cases. By these evaluations, 1896 segments were elaborated. For Technetium-99-m-MIBI SPECT, 1036 segments displayed normal Technetium-99-m-MIBI uptake ( $\geq 70 \%$, classification 2). On the other hand, 860 segments displayed reduced Technetium-99-m-MIBI uptake with different degrees $(<70 \%)$. Elaborations of the latter data, scar tissue with absent Technetium-99-m-MIBI uptake $(\leq 30 \%)$ was imaged in $441(23.2 \%$, classification 0$)$, reduced Technetium-99-m-MIBI uptake from 30\% to $69 \%$ in 419 (22\%, classification 1$)$.

1036 segments with normal Technetium-99-m-MIBI uptake $(\geq 70 \%)$ revealed viability by $\mathrm{F}^{18}$-FDG C-PET/ CT. Predictive value for viability of Technetium-99-mMIBI SPECT therefore presents a value $100 \%$ in this group. Regression analysis revealed a strong linear correlation between segmental $\mathrm{F}^{18}$-FDG and Tc-99m-MIBI uptake with a correlation coefficient of 0.91 $(\mathrm{n}=1036, \mathrm{p}>0.05)$.

Tc-99-m-MIBI uptake between $\geq 30$ and $<70 \%$ was recorded in 419 segments. Corresponding segmental $\mathrm{F}^{18}$ FDG uptake for this group occurred in 347 segments viable myocardial tissue and 72 segments in nonviable $(<50 \%)$. Of details of 347 viable myocardial data from this group, 241 segments revealed a $\mathrm{F}^{18}$-FDG uptake between $\geq 50 \%$ and $<70 \%$. One hundred and six segments were evaluated within $\mathrm{F}^{18}$-FDG uptake $\geq 70 \%$. Predictive value for viability of Technetium-99-m-MIBI SPECT therefore presents a value in $82.8 \%$ in this group. Regression analysis revealed a strong linear correlation between segmental $\mathrm{F}^{18}$-FDG and Tc-99-m-MIBI uptake with a correlation coefficient of $0.79(n=419, p>0.05)$.

Four hundred and forty-one cases with Technetium99-m-MIBI uptake $\leq 30 \%$ (which otherwise would be accepted as poor candidates for revascularization) presented viability with FDG uptakes in 285 cases. One hundred and fifty-six segments from the latter group were verified by FDG uptake as nonviable. The predictive value for Technetium-99-m-MIBI SPECT for this group for viability presents a rate of $35.3 \%$. Regression analysis revealed a weak linear correlation between segmental $\mathrm{F}^{18}$-FDG and Tc-99-m-MIBI uptake with a correlation coefficient of $0.30(n=441, p<0.05)$.

Table 1 depicts elaborate correlation of segmental analysis of Technetium-99-m-MIBI and FDG uptake correction on viability and nonviability.

Classification 1 patients by Tc-99-m-MIBI SPECT ( $\mathrm{n}=419$ segments) were with moderate perfusion findings between $30 \%$ and $70 \%$. In this group, most of the segments revealed viability (347 segments, 82.8\%). However, 71 segments were nonviable by $\mathrm{F}^{18}$-FDG uptake for c-PET/ CT.

Classification 0 patients were with severe perfusion defects lower than $30 \%$ in 441 segments by Tc-99-mMIBI SPECT. These patients were poor candidates for revascularization. $\mathrm{F}^{18}$-FDG uptake for c-PET/CT among this group presented similar results in 156 segments

Table 1. Segmental comparison of Tc-99-m-MIBI and corresponding FDG uptake

\begin{tabular}{l|l}
$\begin{array}{l}\text { Tc-99-m-MIBI uptake, } \\
\text { segments }\end{array}$ & Corresponding F18-FDG uptake \\
\hline$<30 \%, 441$ (classification 0) & $\geq 50-70 \%, 285$ (viable) \\
& $<50 \%, 156$ (nonviable) \\
& $>70 \%, 106$ (viable) \\
\hline $\begin{array}{l}\geq 30 \text { and }<70 \%, 419 \\
\text { (classification 1) }\end{array}$ & $\geq 50-70 \%, 241$ (viable) \\
\hline $\begin{array}{l}\geq 70 \%, 1036 \text { (classification 2) } \\
\text { MIBI: Myocardial perfusion imaging test, FDG: Fluorodeoxyglucose }\end{array}$ & $>70 \%, 1036$ (nonviable) \\
\hline $\begin{array}{l}\text { All classification 2 patients with normal perfusion by TC-99-m-MIBI SPECT } \\
\text { (n=1036 segments) presented viability with F18-FDG cardiac PET/CT } \\
\text { images }\end{array}$
\end{tabular}


(35.3\%) with nonviable myocardial tissue. On the contrary to Tc-99-m-MIBI SPECT, 285 segments $(64.5 \%)$ proved viability by $\mathrm{F}^{18}$-FDG uptake for c-PET/CT.

Figure 1. Depicts the myocardial viability segments corrections by $\mathrm{F}^{18}$-FDG uptake for c-PET/CT versus Tc99-m MIBI uptake results.

Segments from classification 2 were identical between Tc-99-m MIBI SPECT and FDG PET/CT for perfusion and viability. Statistically strongest discorrelation was observed in classification $0(\mathrm{p}<0.05)$.

Figure 2. Coronary lesions from coronary angiography demonstrates a certain indication for $\mathrm{CABG}$ for at least three vessel surgeries.

Figure 3. This patient was from Classification $\mathrm{O}$ by Tc-99-m MIBI SPECT with perfusion less than 30\%. Anterior wall mid-apical segments, inferior wall and apex were reported as infarct with fixed a perfusion which was accepted as scar tissue. The secondary correcting $\mathrm{F}^{18}$ FDG uptake for c-PET/CT evaluation was also reported

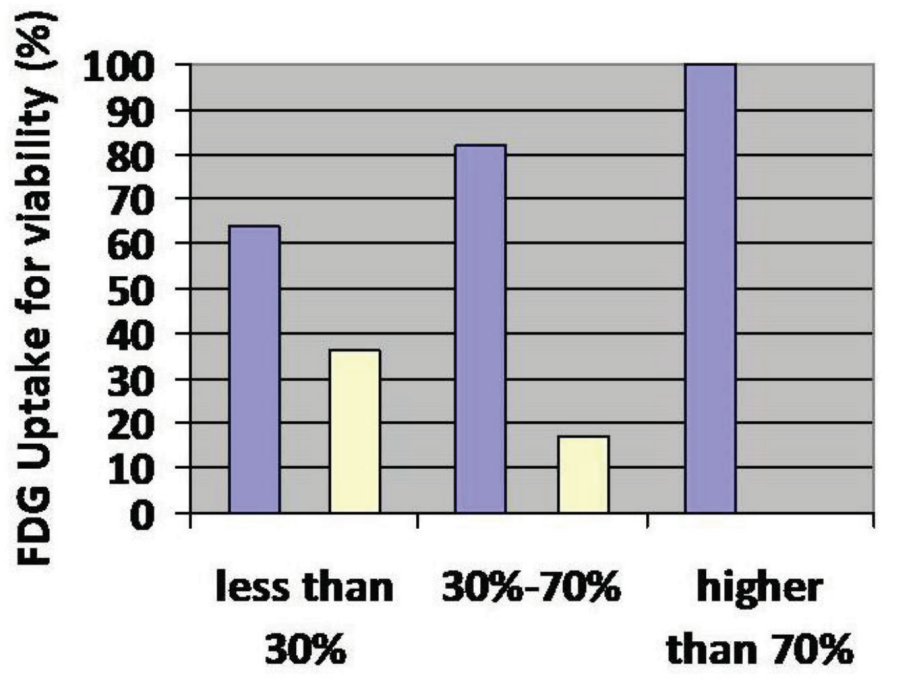

Tc-99-m MIBI Uptake

\section{viable $\square$ Nonviable}

Figure 1. Correction of Tc-99-m MIBI SPECT by FDG PET/CT for viability

MIBI: Myocardial perfusion imaging test, FDG: Fluorodeoxyglucose, PET: Positron emission tomography, CT: Computed tomography as anterior wall, anteroapical segment, apex, apicolateral segment, inferior wall and inferoapical segments without metabolic activity. The latter statement was identical to Tc99-m MIBI SPECT results as scar tissue with nonviable myocardium. This patient was not operated due to nonbeneficial myocardial conditions for revascularization.

Figure 4. Conventional coronary angiography reveals a need for revascularization for both left and right coronary system.

Figure 5. This patient was also from Classification $\mathrm{O}$ by Tc-99-m MIBI SPECT with perfusion less than 30\%. Anterior wall, anteroseptal wall mid-apical segments, apex, inferioapical and inferolateral segments were reported as infarct with fixed no-perfusion which was accepted as scar tissue. At this point, without a secondary evaluation, this case was not a candidate for revascularization. However, the secondary correcting $\mathrm{F}^{18}$-FDG uptake for c-PET/ $\mathrm{CT}$ evaluation was reported as hypometabolism from inferolateral wall mid-basal segments and septal regions of inferoapical segments. This report was accepted as hibernating myocardial tissue. Other segments of left ventricle were within normal metabolic activity with strong FDG uptake. These segments were accepted as viable myocardial tissue. This cardiac PET/CT statement was not similar and/or parallel to Tc-99-m MIBI SPECT results. This patient was operated due to strong evidences of myocardial viability and hibernating myocardium. We were able to discharge this case after an uneventful postoperative period.

\section{Discussion}

We believe that $\mathrm{CABG}$ can be performed with feasible and acceptable postoperative morbidity and mortality rates in patients with ischemic left ventricular dysfunction ${ }^{(1-3)}$. Pocar et al. ${ }^{(4)}$ reported that viability testing had an established role in the identification of patients who were unlikely to benefit from revascularization. In parallel with our opinion, this author believed that the extent of viability was also shown to predict reverse ventricular remodelling. Data concerning survival beyond 5 years 
scarce, but reports addressing ventricular dysfunction often include patients with concurrent angina. Conclusion from Pocar et al. ${ }^{(4)}$ study reporting a retrospective analysis for long term survival beyond 10 years can be anticipated in patients with advanced ischemic heart failure and residual viability, irrespective of concurrent angina. Pocar

\section{Left system}

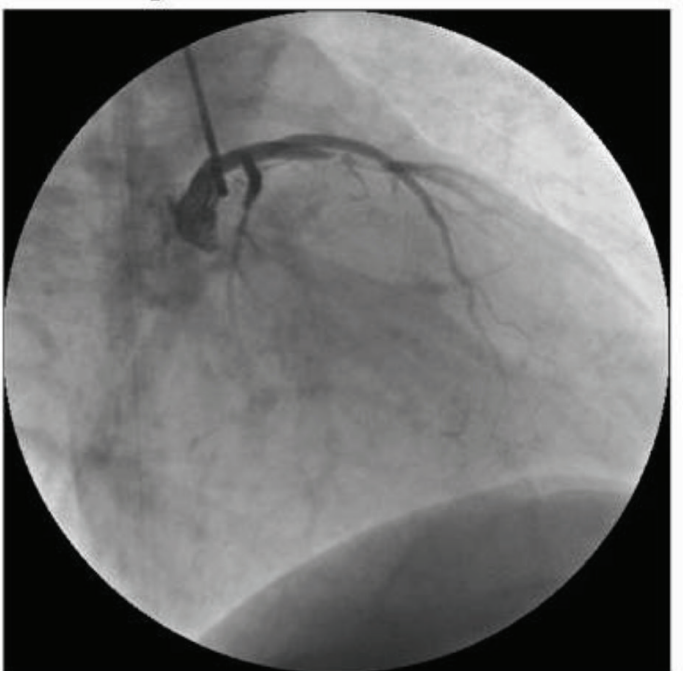

Figure 2. Coronary angiography case 1

\section{Tc 99-m-MIBI SPECT}
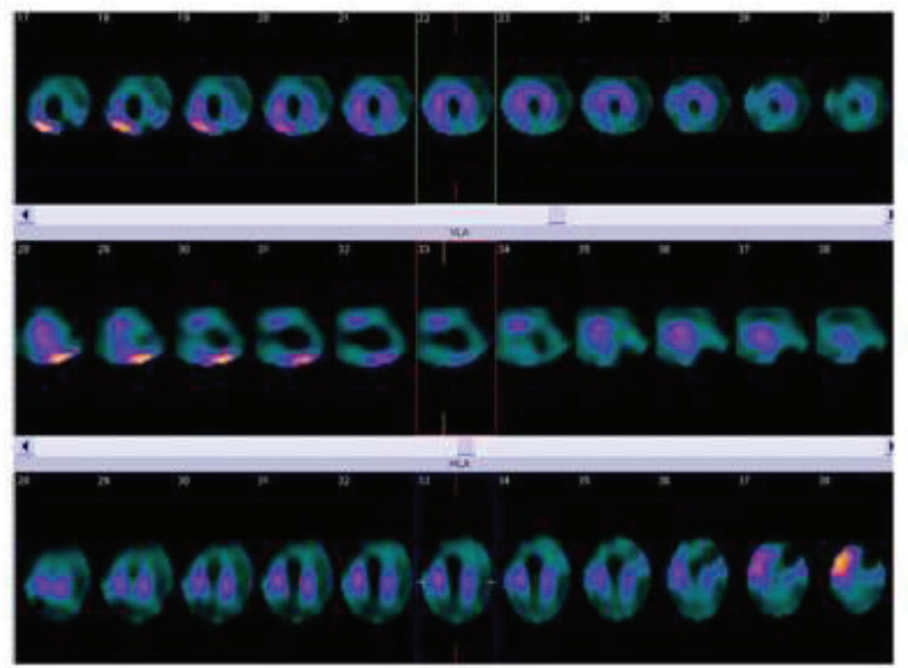

Figure 3. MIBI and PET/CT of case 1

MIBI: Myocardial perfusion imaging test, FDG: Fluorodeoxyglucose, PET: Positron emission tomography, CT: Computed tomography, SPECT: Single photon emission computed tomography

Talay and Belgit Talay. Preoperative Correction of Tc-99-m-MIBI SPECT by F18-FDG Cardiac PET/CT for Myocardial Viability Before CABG

\section{FDG cardiac PET-CT}

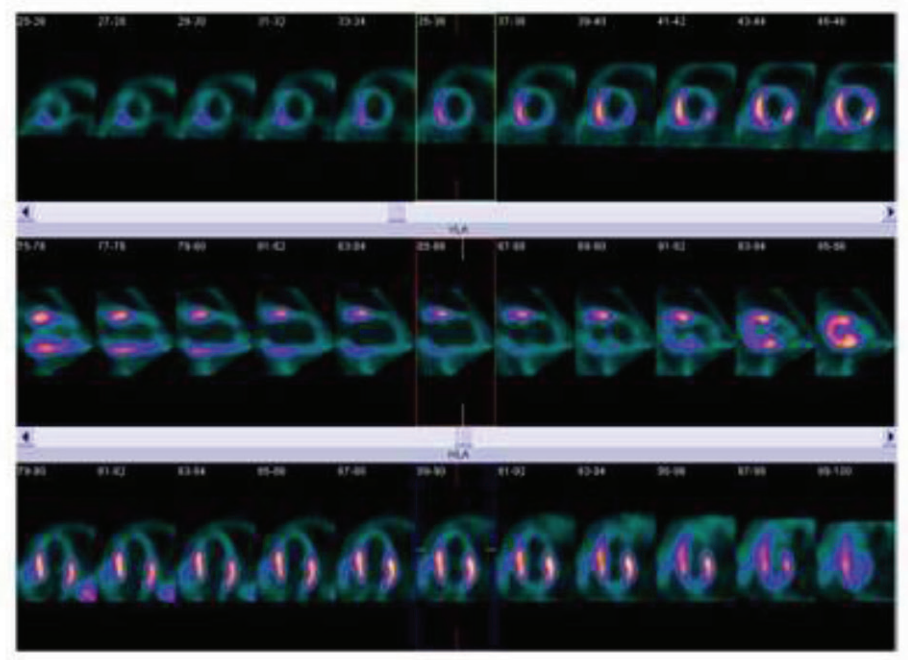

et al. ${ }^{(4)}$ probability of survival at 15 years for their entire cohort was $44 \%$, which was up to $51 \%$ in patients with a preoperative left ventricular end diastolic volume of less than $25 \mathrm{mmHg}$. We did not evaluate preoperative left ventricular end diastolic volume independently in our study; however, this entity may be correlated with higher

\section{Right system}

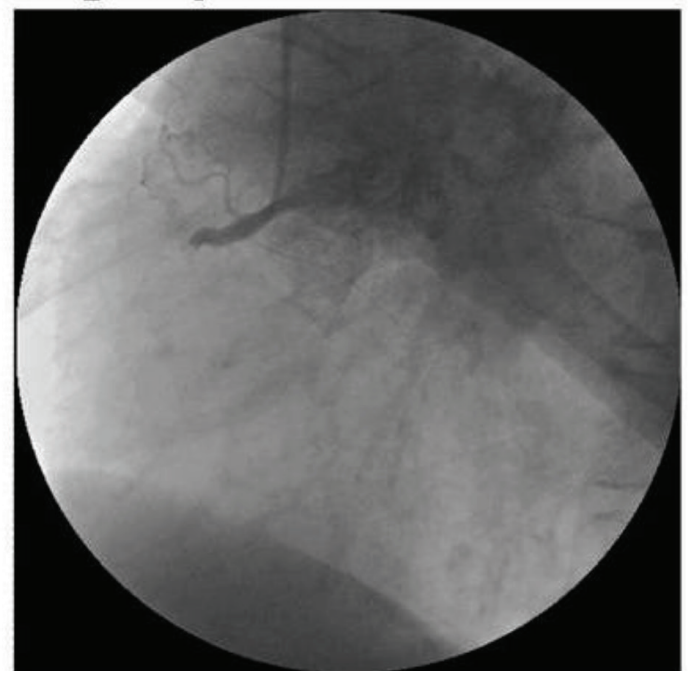


mortality as reported by Pocar et al. ${ }^{(4)}$. On the contrary, Pocar et al. ${ }^{(4)}$ advocated cardiac transplantation, which we believe to be the gold standard for these group of patients, and it might be with slightly better long-term outcomes when compared to CABG. Nevertheless, from our point of view, preoperative existence of cardiac viability and its determination play the single utmost important key

\section{Left system}

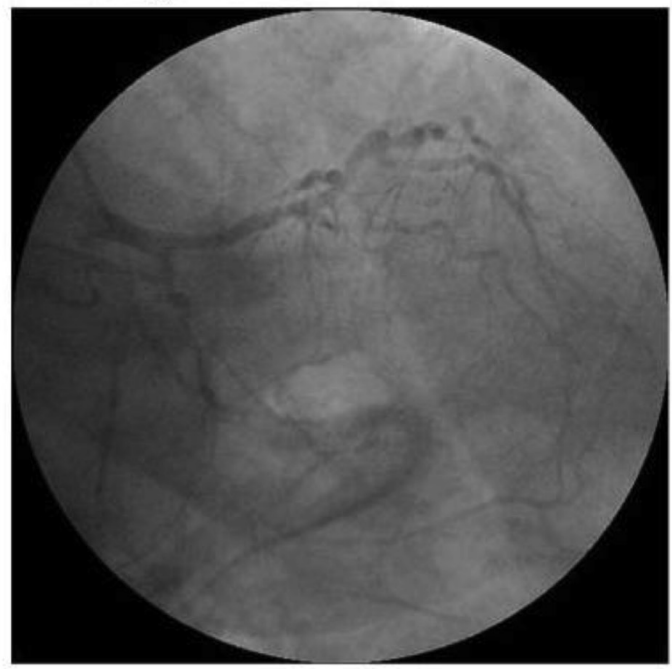

Figure 4. Coronary angiography case 2

\section{Tc 99-m-MIBI SPECT}
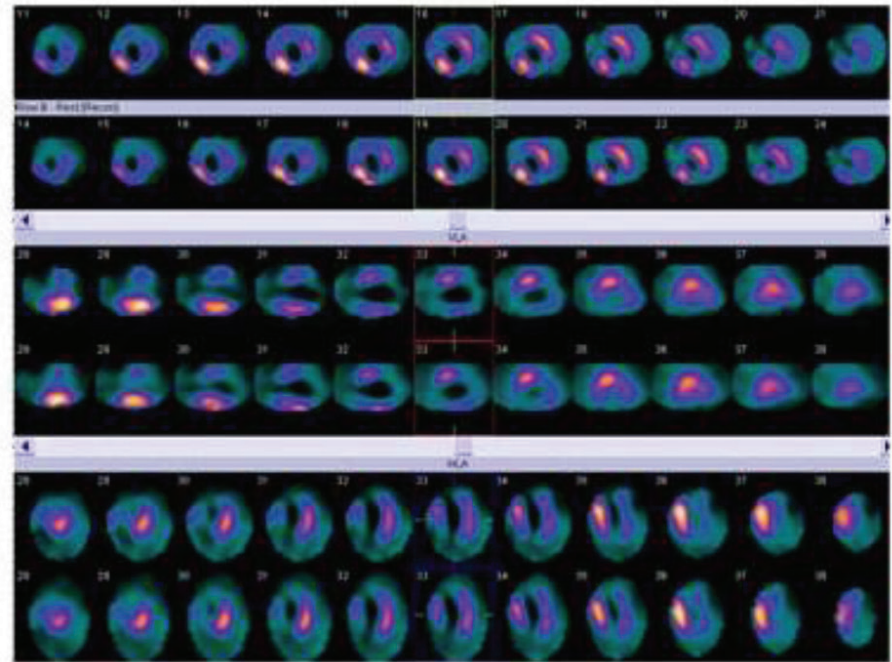

role for postoperative survival ${ }^{(5-7)}$. At this point, it is crucial to decide which technique is to be with more exact determination $^{(8,9)}$.

An increased risk of perioperative mortality has been observed in lower LVEF group in our study. Re-do $\mathrm{CABG}$, the only other independent predictor of mortality, was observed in 10 cases among 18 in total. Nevertheless,

\section{Right system}

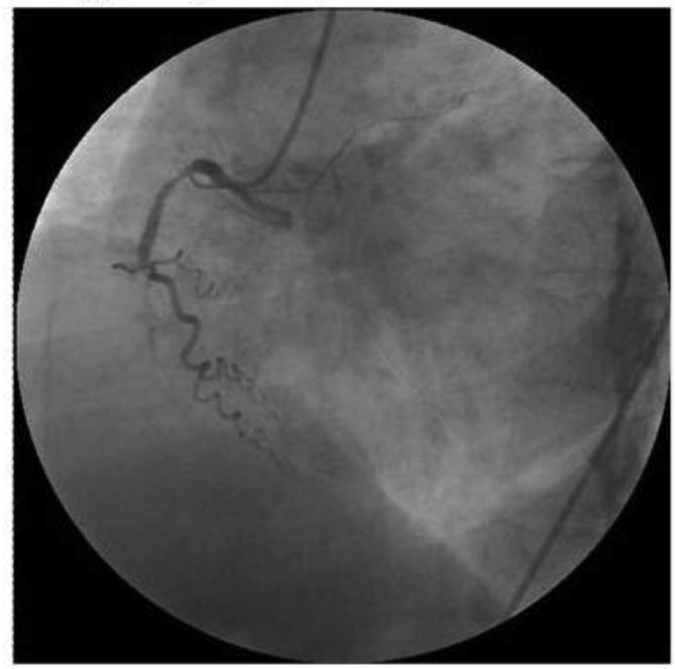

\section{FDG cardiac PET-CT}

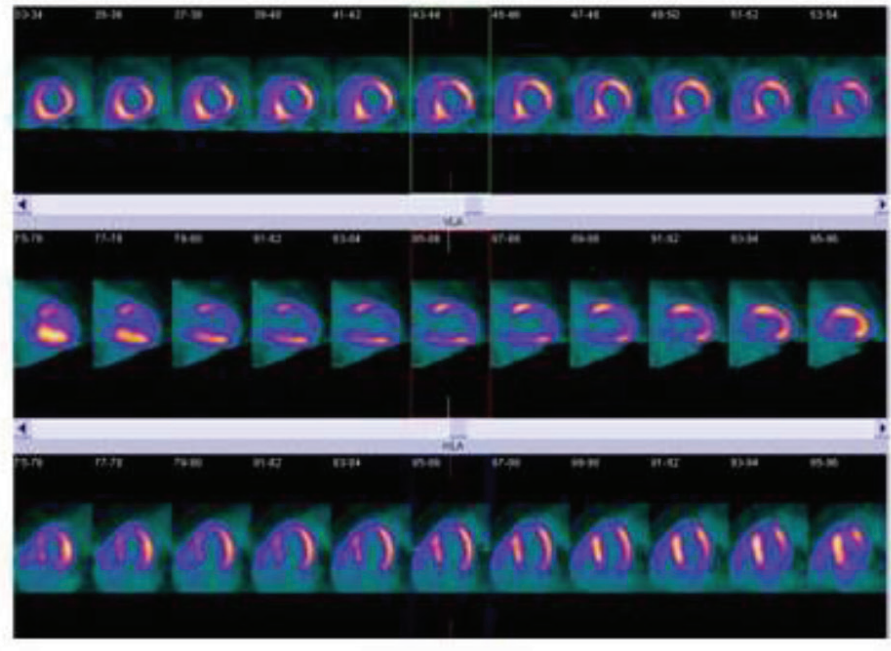

Figure 5. MIBI and PET/CT case 2

MIBI: Myocardial perfusion imaging test, FDG: Fluorodeoxyglucose, PET: Positron emission tomography, CT: Computed tomography, SPECT: Single photon emission computed tomography 
these mortality considerations stress the importance of patient selection, suggesting that mortality and morbidity risk might be encountered by strong possibilities of postoperative left ventricular recovery in function which can be achievable through demonstrated existence of viable myocardial segments in the left ventricle ${ }^{(10)}$.

In this context, the aim of our study was not to determine survival and/or perioperative predictors of mortality and morbidity. We simply targeted to compare Technetium-99-m-MIBI SPECT and $\mathrm{F}^{18}$-FDG Cardiac $\mathrm{PET} / \mathrm{CT}$ for myocardial viability testing.

From our point of view, in parallel with medical publications especially from nuclear medicine working field $^{(11-14)}, \mathrm{F}^{18-}$ FDG cardiac PET/CT is with higher accuracy and sensitivity for viability detection when compared to other techniques such as technetium SPECT and dobutamine stress echocardiographic tests. Nevertheless, Tc-99-m-MIBI SPECT is a valuable technique to evaluate perfusion.

Saghari et al. ${ }^{(15)}$ evaluated the frequency and severity of myocardial perfusion abnormalities using Tc-99-m MIBI SPECT in cardiac syndrome X. In this study, 36 patients were evaluated for perfusion analysis in possible defects. Perfusion degree was divided into three main classifications; mild, moderate and severe. Saghari et al. ${ }^{(15)}$ was also able to determine the relevant coronary regions. Of these 36 patients, 13 (36.1\%) had a normal Tc-99-m MIBI SPECT study, 23 (63.9\%) had an abnormal Tc-99-m MIBI SPECT study. Their study suggested that the severity of perfusion defects among patients with coronary artery stenosis could be evaluated by Tc-99-m MIBI SPECT, effectively.

A comparison study between Tc-99-m MIBI SPECT and $\mathrm{F}^{18}$-FDG PET was reported by Altehoefer et al. ${ }^{(16)}$. They signed out that the pathophysiological significance of Tc-99-m MIBI SPECT uptake at rest for assessing myocardial viability with coronary artery disease was controversial. They studied the relationship of Tc-99-m MIBI SPECT uptake at rest and preserved or absent uptake of FDG assessed with PET in 111 consecutive patients. As a similar module to our study algorithm, they evaluated each ventricle in 13 segments which were derived from 25 regions of interest (ROI) in short axis cuts and normalized the FDG uptake to the intraindividual normal reference ROI (ROI with maximal $=100 \%$ Tc-99-m MIBI uptake). Altehoefer et al. ${ }^{(16)}$ accepted normalized segments with FDG uptake higher than $70 \%$ as viable and segments with FDG uptake less than 50\% as nonviable. Their study results concluded that 5 to $11 \%$ of segments with Tc-99-m MIBI uptake at rest $\leq 30 \%$ of peak activity were viable and $80-84 \%$ nonviable. Of moderate to severe Tc- $99-\mathrm{m}$ MIBI defects at rest (31-70\% of peak), $13-61 \%$ were viable. Segmental Tc-99-m MIBI uptake and normalized FDG uptake were reported to be linearly correlated. Altehoefer et al. ${ }^{(16)}$ concluded that in patients with coronary artery disease, Tc-99-m MIBI uptake underestimated myocardial viability in comparison to FDG PET. This statement was identical with ours. Furthermore, they also advocated that myocardial Tc-99-m MIBI uptake appeared to reflect myocardial blood flow rather than myocardial viability. Patients with moderate and severe Tc-99-m MIBI defects at rest may benefit from additional metabolic PET imaging prior to final therapeutic decisions. These myocardial dynamics are especially important for borderline patient with severely reduced LVEF for final surgical decisions ${ }^{(17,18)}$. We believe that it is mandatory to state under these findings that a surgical decision solely depending on Tc-99-m MIBI would be inadequate. As a conventional reflex, the majority of surgeons administer to Tc-99-m MIBI to evaluate the feasibility of a CABG for lower LVEF cases. However, defects on perfusion by Tc-99-m MIBI may not necessarily present the viability of myocardium. Because it is a well-known fact that hypoperfusion and/or a perfusion images from Tc-99-m MIBI may also be presenting hibernating and/or periinfarct ischemia regions. A second point is that these latter segments may clearly benefit from revascularization by functional recoveries at the postoperative period.

Therefore, our results also provide information about the effectiveness of $\mathrm{F}^{18}$-FDG C-PET/CT on understanding 
myocardial metabolic kinetics by concerns of viability detection. $\mathrm{F}^{18}$-FDG C-PET/CT clearly enlighten the borderline situations with suspicious Tc-99-m MIBI negative myocardium by perfusion defects ${ }^{(19)}$. At this point, we advocate Tc-99-m MIBI SPECT to be handicapped to elaborate myocardial viability and indications of $\mathrm{CABG}$ when compared to $\mathrm{F}^{18}$-FDGC-PET/CT for lower LVEF cases. Especially, cases of classification 0 with severe perfusion defects and classification 1 with moderate perfusion defects by Tc-99-m MIBI SPECT must be evaluated by $\mathrm{F}^{18}$-FDG C-PET/CT for myocardial viability prior to finalize the surgical decision. On the other hand, a relatively normal perfusion results by Tc-99-m MIBI SPECT may clarify the suspicious myocardial conditions well-enough to decide for surgery. From our study results, the latter group is to be with $100 \%$ correlation $\mathrm{F}^{18}$-FDG C-PET/CT. So, for this positive MIBI SPECT cases, we believe that there is no need to seek for secondary evidence for the "obvious" condition prior to surgery by cardiac PET/CT.

Long-term results and survival studies to investigate for patients who were operated via $\mathrm{F}^{18}$-FDG C-PET/CT results are another subject for further researches. The aim of this recent study was to determine the difference between $\mathrm{F}^{18}$-FDG C-PET/CT and Tc-99-m-MIBI SPECT techniques on preoperative myocardial viability detection.

\section{Conclusion}

Prior to $\mathrm{CABG}$ surgery decision with reduced preoperative LVEF, cardiac PET/CT using $\mathrm{F}^{18}$-FDG predicts myocardial viability more effectively when compared to Tc-99-m-MIBI SPECT alone. After a report of a severe hypoperfusion by Tc-99-m-MIBI SPECT analysis, it is suggestible to apply $\mathrm{F}^{18}$-FDG cardiac PET/ $\mathrm{CT}$ as a last resort. We believe that abandoning surgery by Tc-99-m-MIBI SPECT is a premature decision.

Peer-review: Externally peer-reviewed.

\section{Authorship Contributions}

Surgical and Medical Practices: S.T., N.B.T., Concept: S.T., N.B.T., Design: S.T., N.B.T., Data Collection or
Processing: S.T., N.B.T., Analysis or Interpretation: S.T., N.B.T., Literature Search: S.T., N.B.T., Writing: S.T., N.B.T.

Conflict of Interest: No conflict of interest was declared by the authors.

Financial Disclosure: The authors declared that this study received no financial support.

\section{References}

1. Hassanabad AF, MacQueen KT, Ali I. Surgical Treatment for Ischemic Heart Failure (STICH) trial: A review of outcomes. J Card Surg 2019;34:1075-82.

2. Yu PJ, Lin D, Catalano M et al. Predictors of Increased Length of Hospital Stay in Patients with Severe Cardiomyopathy Undergoing Coronary Artery Bypass Grafting. J Cardiothorac Vasc Anesth 2019;33:2703-8.

3. Mickleborough LL, Carson S, Tamariz M et al. Results of revascularization in patients with severe left ventricular dysfunction. J Thorac Cardiovasc Surg 2000;119:550-7.

4. Pocar M, Di Mauro A, Passolunghi D, et al. Predictors of adverse events after surgical ventricular restoration for advanced ischaemic cardiomyopathy. Eur J Cardiothorac Surg 2010;37:1093-100.

5. Topuz U, Salihoglu Z, Gokay BV, et al. The effects of different oxygen concentrations on recruitment maneuver during general anesthesia for laparoscopic surgery. Surg Laparosc Endosc Percutan Tech 2014;24:410-3.

6. He XY, Gao CQ. Peri-operative application of intra-aortic balloon pumping reduced in-hospital mortality of patients with coronary artery disease and left ventricular dysfunction. Chin Med J (Engl) 2019;132:935-42.

7. Günel SE, Akgun A. Comparison of exercise-rest-reinjection Tl-201 imaging and rest sublingual isosorbide dinitrate Tc-99m MIBI imaging for the assessment of myocardial viability. Ann Nucl Med 2009;23:451-7.

8. Slart RH, Bax JJ, van Veldhuisen D, et al. Prediction of functional recovery after revascularization in patients with chronic ischaemic left ventricular dysfunction: head-to-head comparison between $99 \mathrm{mTc}$-sestamibi/18FFDG DISA SPECT and 13N-ammonia/ 18F-FDG PET. Eur J Nucl Med Mol Imaging 2006;33:716-23.

9. Gunning MG, Chua TP, Harrington D, et al. Underwood SR. Hibernating myocardium: clinical and functional response to revascularization. Eur J Cardio-thorac Surg 1997;11:1105-12.

10. Lee MS, Park HS, Lee BC, et al. Identification of Angiogenesis Rich-Viable Myocardium using RGD Dimer based SPECT after Myocardial Infarction. Sci Rep 2016;6:27520

11. Canty JM Jr, Fallavollita JA. Chronic hibernation and chronic stunning: a continuum. J Nucl Cardiol 2000;7:509-27.

12. La Canna G, Rahimtoola SH, Visioli O, et al. Sensitivity, specificity, and predictive accuracies of non-invasive tests, singly and in combination, for diagnosis of hibernating myocardium. Eur Heart J 2000;21:1358-67.

13. Inaba Y, Chen JA, Bergmann SR. Quantity of viable myocardium required to improve survival with revascularization in patients with ischemic cardiomyopathy: A meta-analysis. J Nucl Cardiol 2010;17:646-5. 
14. Benz DC, von Dahlen AP, Huang W, et al. No differences in rest myocardial blood flow in stunned and hibernating myocardium: insights into the pathophysiology of ischemic cardiomyopathy. Eur J Nucl Med Mol Imaging 2019;46:2322-8

15. Saghari M, Assadi M, Eftekhari M, et al. Frequency and severity of myocardial perfurion abnormalities using Tc-99-m MIBI SPECT in cardiac syndrome x. BMC Nucl Med 2006;6:1.

16. Altehoefer C, vom Dahl J, Biedermann M. Significance of defect severity in Technetium-99m MIBI SPECT at rest to assess myocardial viability: comparison with Fluorine-18-FDG PET. J Nucl Med 1994;35:569-74.

17. Glaveckaite S, Valeviciene N, Palionis D, et al. Prediction of long-term segmental and global functional recovery of hibernating myocardium after revascularisation based on low dose dobutamine and late gadolinium enhancement cardiovascular magnetic resonance. J Cardiovasc Magn Reson 2014;16:83.

18. Romero J, Lupercio F, Haramati LB, et al. Myocardial viability and microvascular obstruction: role of cardiac magnetic resonance imaging. Cardiol Rev 2014;22:246-52.

19. Cohade C, Wahl RL. Applications of positron emission tomography/ computed tomography image fusion in clinical positron emission tomography: clinical use, interpretation methods, diagnostic improvements. Semin Nucl Med 2003;33:228-37. 